\title{
Coherent detection of the light backscattered by an optical surface
}

Imran Khan, Michel Lequime, Myriam Zerrad, Claude Amra

Imran Khan, Michel Lequime, Myriam Zerrad, Claude Amra, "Coherent detection of the light backscattered by an optical surface," Proc. SPIE 11852, International Conference on Space Optics - ICSO 2020, 1185241 (11 June 2021); doi: 10.1117/12.2599635

SPIE Event: International Conference on Space Optics - ICSO 2021, 2021, Online Only 


\section{International Conference on Space Optics-ICSO 2020}

Virtual Conference

30 March-2 April 2021

Edited by Bruno Cugny, Zoran Sodnik, and Nikos Karafolas
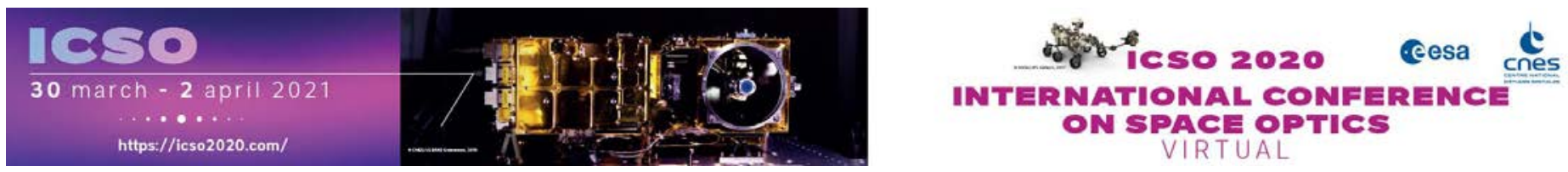

Coherent detection of the light backscattered by an optical surface

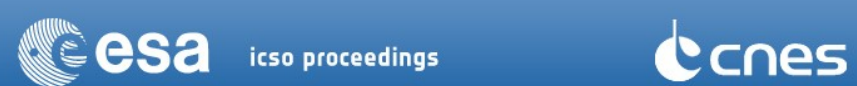




\title{
Coherent Detection of the Light Backscattered by an Optical Surface
}

\author{
Imran Khan, Michel Lequime, Myriam Zerrad, and Claude Amra \\ Aix Marseille Univ, CNRS, Centrale Marseille, Institut Fresnel, Marseille, France
}

\begin{abstract}
We measured very low light power backscattered by optical surfaces using low coherence interferometry. The backscattered light can indeed be a critical noise source for interferometric gravitational wave detectors such as LISA, the laser interferometer space antenna developed by ESA, with the support of NASA and European national space agencies. We present the preliminary measurement results of light backscattered from a silver mirror and a bare N-BK7 window $1 \mathrm{~mm}$ thick using a superluminescent diode centered at $1060 \mathrm{~nm}$ as broadband light source. The use of low coherence interferometry allows light backscattered by both sides of the N-BK7 window to be recorded independently with a noise floor of around $-110 \mathrm{~dB}$.
\end{abstract}

Keywords: Scattered Light, Low Coherence Interferometry, Gravitational Waves Detector

\section{INTRODUCTION}

With the first direct detection of Gravitational Waves (GW) by LIGO and Virgo scientific collaboration [1] in September 2015, a new observational astronomy window on the universe has been established. And the network of LIGO [2] and Virgo [3] detectors have reported numerous number of GW events consisting of binary black hole systems, a neutron star and black hole system, and rotating neutron star system $[4,5,6]$ with the heaviest GW event detected so far of total mass of $150 \mathrm{M}_{\circ}$ [7]. The current generation of $\mathrm{GW}$ detectors and future planned third generation (Einstein Telescope, ET) will further improve the strain sensitivity in the detection bandwidth of $1 \mathrm{~Hz}$ to $10 \mathrm{kHz}$, thus increasing the detection efficiency of GW sources of mass in the range of stellar mass black holes (few 100's of $\mathrm{M}_{\circ}$ ), rotating neutron stars and and asymmetric supernovae sources.

However, to explore the GW sources emitting at low frequency in the bandwidth $(0.1 \mathrm{mHz}$ to $1 \mathrm{~Hz})$, that correspond to astrophysical sources such as super massive black holes and dense stars captured by super massive black holes, an interferometer with very long arms is required. Due to the seismic activity of the Earth such large scale interferometer cannot be built on earth and thus, the outer space becomes the ultimate choice. One such large scale interferometer, named as Laser Interferometer Space Antenna (LISA) that will have interferometer arm lengths on the order of millions of kilometers was proposed by ESA with the help of NASA and European national space agencies and is currently under the initial phase of development $[8,9,10]$.

For all these GW interferometric instruments, parasitic light $[10,11,12,13,14]$ is a critical noise source because, as a first order approximation, it can induce phase measurement errors depending on the relative amplitude of these parasitic optical fields. By way of example, this means that phase uncertainties of the order of a few microradians can be induced by ultra-weak retroreflected or back-scattered light, the relative power of which is about $10^{-12}$. The availability of a characterization equipment capable of detecting such ultra-low light power is therefore a key point of the development of this new generation of GW detectors.

Further author information: (Send correspondence to Imran Khan and Michel Lequime)

Imran Khan: E-mail: imran.khan@fresnel.fr,

Michel Lequime: E-mail: michel.lequime@fresnel.fr 


\section{THEORETICAL BACKGROUND}

Low coherence interferometry is a powerful tool in precise measurements of surface details of samples, and in particular the characterization of samples with multiple surfaces $[15,16]$. The basic principle consists of a broadband light beam that is split into the two arms of a Michelson interferometer i.e, the reference and the sample arms, and the resulting signal due to the mixing of light backreflected by the two arms is detected using a photodiode.

The output signal of the interferometer depends on the amount of light power back-reflected by each of the two arms (with surface reflectivities $R_{r}$ and $R_{s}$ ) and the optical path difference $\Delta=2\left(z_{r}-z_{s}\right)$ between these two arms. Mathematically, the output signal for a single optical interface is given by

$$
S\left(z_{r}, z_{s}\right)=S_{0}\left(R_{r}+R_{s}+2 \sqrt{R_{r} R_{s}} e^{-(\pi \delta \sigma \Delta)^{2}} \cos k_{0} \Delta\right)
$$

where $S_{0}$ is the spectrally integrated power of the source, here assumed to be of Gaussian shape, $k_{0}$ the central

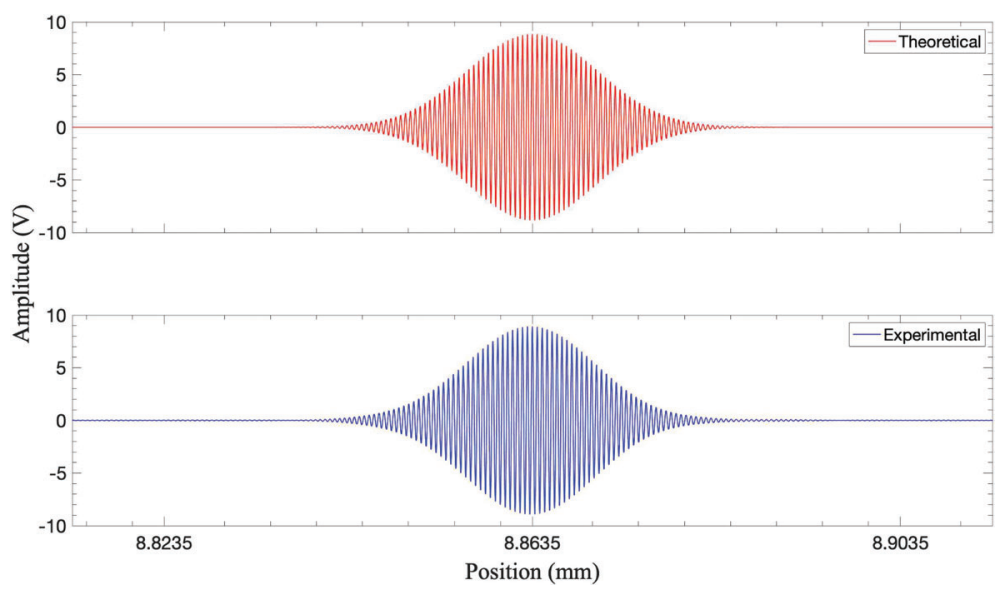

Figure 1: Theoretical versus experimentally measured low coherence interferometric signal for a source at $\lambda_{0}=1060 \mathrm{~nm}$ with optical $3 \mathrm{~dB}$ bandwidth of around $70 \mathrm{~nm}$.

wave vector, and $\delta \sigma$ the source bandwidth in wavenumber. Consequently, the signal $S$ is only modulated around zero optical path difference (OPD) and over a characteristic distance $l_{c}$ named the coherence length of the source and defined by

$$
l_{c}=\frac{1}{\pi \delta \sigma}=\frac{2 \sqrt{\ln (2)}}{\pi} \frac{\lambda_{0}^{2}}{\Delta \lambda}
$$

where $\lambda_{0}$ is the central wavelength of the source and $\Delta \lambda$ its spectral full width at half maximum (FWHM). Figure 1 shows a comparison between the theoretical shape of the modulated part of the interferometric signal $\left(\lambda_{0}=1060 \mathrm{~nm}, \Delta \lambda=70 \mathrm{~nm}\right)$ and the one experimentally recorded. For a multiple reflections sample, the low coherence interferometric signal is a function of the length of the scanned reference arm and given by

$$
S\left(z_{r}\right)=S_{0}\left(R_{r}+\sum_{m=1}^{N} R_{s, m}+2 \sum_{n=1}^{N} \sqrt{R_{r} R_{s, m}} e^{-\left[2 \pi \delta \sigma\left(z_{r}-z_{s, m}\right)\right]^{2}} \cos \left[2 k_{0}\left(z_{r}-z_{s, m}\right)\right]\right)
$$

where $m$ is an integer that defines the order of the back-reflected signal. The scan of the reference arm allows the successive reflections (echoes) to be independently recorded and quantified. As an example, if a 1-mm thick N-BK7 window is installed in the sample arm of the Michelson interferometer at zero angle of incidence, we are capable to successively record the reflection on the front face $\left(R_{s, 1}=0.04, z_{r}=z_{s}\right)$, the reflection on the rear face $\left(R_{s, 2}=0.037, z_{r}=z_{s}+n d, d=1 \mathrm{~mm}\right.$ and $\left.n=1.5054\right)$, the first multiple reflection within the window $\left(R_{s, 3}=6.2 \times 10^{-5}, z_{r}=z_{s}+2 n d\right)$, the second multiple reflection within the same window $\left(R_{s, 4}=\right.$ $\left.1.02 \times 10^{-7}, z_{r}=z_{s}+3 n d\right)$, and so on $\left[R_{s, m}=(1-R)^{2} R^{(2 m-3)}, z_{r}=z_{s}+(m-1) n d, m \geq 2\right]$ up to the limit defined by the noise floor of the set-up. 


\section{EXPERIMENTAL SETUP}

The optical layout of the experiment is shown in Figure 2a and a picture of the implemented bench is shown in Figure 2b. Our experiment consists of a $60 \mathrm{~mW}$ pigtailed superluminescent diode (SLD) with a central wavelength of $1060 \mathrm{~nm}$ and FWHM bandwidth of $70 \mathrm{~nm}$. The pigtail consists of a Corning HI 780 single mode fiber whose output is located in the focal plane of a reflective collimator with an effective focal length of $7 \mathrm{~mm}$. This assembly provides a Gaussian collimated light beam with a waist diameter around $2 \mathrm{~mm}$. The input optical beam is split using a polarizing cube beam splitter (BS1) into the signal and reference arms of the interferometer and the split ratio can be adjusted by changing the orientation of the linear polarizer P0. The beam reflected by the polarizing beam splitter (S polarization) corresponds to the sample arm. It crosses a quarter wave plate with axis at 45 degrees and illuminates the sample whose tilt angle is $\theta$. The light back-reflected $(\theta=0)$ or back-scattered $(\theta>0)$ by the sample is transformed in a P-polarized beam by the back-crossing of the quarter wave plate, then is transmitted by the polarizing beam splitter.

The beam transmitted by the polarizing beam splitter ( $\mathrm{P}$ polarization) corresponds to the reference arm

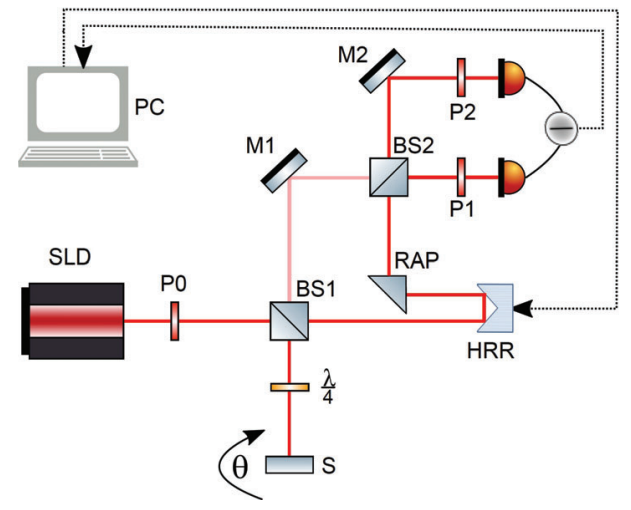

(a) Layout of the optical bench.

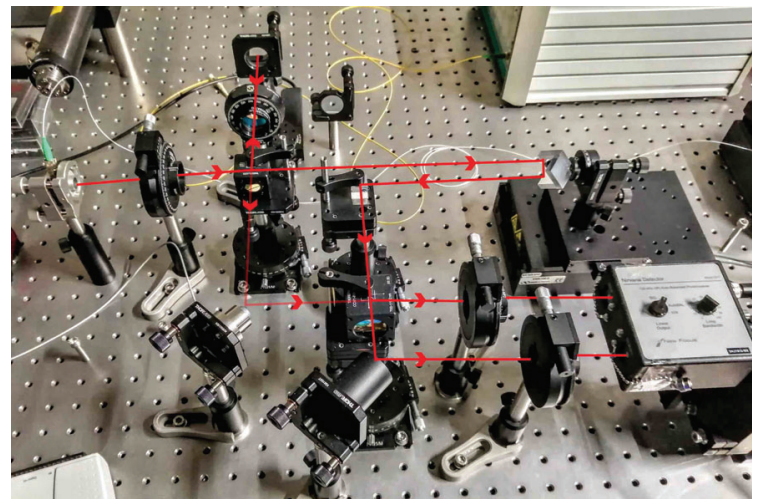

(b) The implemented laboratory bench.

Figure 2: The experiment.

and is reflected by a hollow retroreflector (HRR). The HRR along with a right angle prism (RAP) allows the reflected beam to be decoupled from the incidence path such that it can be mixed with the signal. The hollow retroreflector is mounted on a Newport XMS-100 S motorized linear translation stage, while the sample is mounted on a Thorlabs PRMTZ8 motorized rotation. The sample beam and the reference beam are mixed on a non-polarizing beam splitter (BS2) and detected using a balanced receiver including two InGaAs photodiodes (Newport Nirvana 2017 model). Two linear polarizers (P1 and P2) are used to optimize the balance between the DC part of the currents provided by each photodetector. The output channel of the balanced detection is acquired using National Instruments (NI) data acquisition (DAQ) module USB-6212 (16 bits, $100 \mathrm{kSamples} / \mathrm{s}$ ) and post processed numerically.

\section{RESULTS AND DISCUSSION}

We measured light backscattered by two different samples i.e, $1 \mathrm{~mm}$ thick N-BK7 optical window and a Silver mirror. In order to demonstrate the sensitivity of the experimental set-up, we first measured the light backreflected from a $1 \mathrm{~mm}$ thick N-BK7 window at zero AOI.

The reference arm is scanned at a constant velocity of $0.5 \mathrm{~mm} / \mathrm{s}$, which generates a carrier frequency of around $935 \mathrm{~Hz}$ in the signal resulting from the differential amplification of the photodiode outputs. A bandpass filtering is implemented on this acquired signal (bandwidth $50 \mathrm{~Hz}$ ) and allows the noise level to be drastically reduced. A key point of this balanced detection scheme is the necessity to avoid any saturation of the two photodetectors. This implies to limit the overall detected power (DC plus AC parts) below $0.5 \mathrm{~mW}$. For instance, in the case of the detection of multiple reflections on a N-BK7 window, we operated the SLD source below $2 \mathrm{~mW}$ of continuous power (driving current $180 \mathrm{~mA}$ ) to meet this condition. 

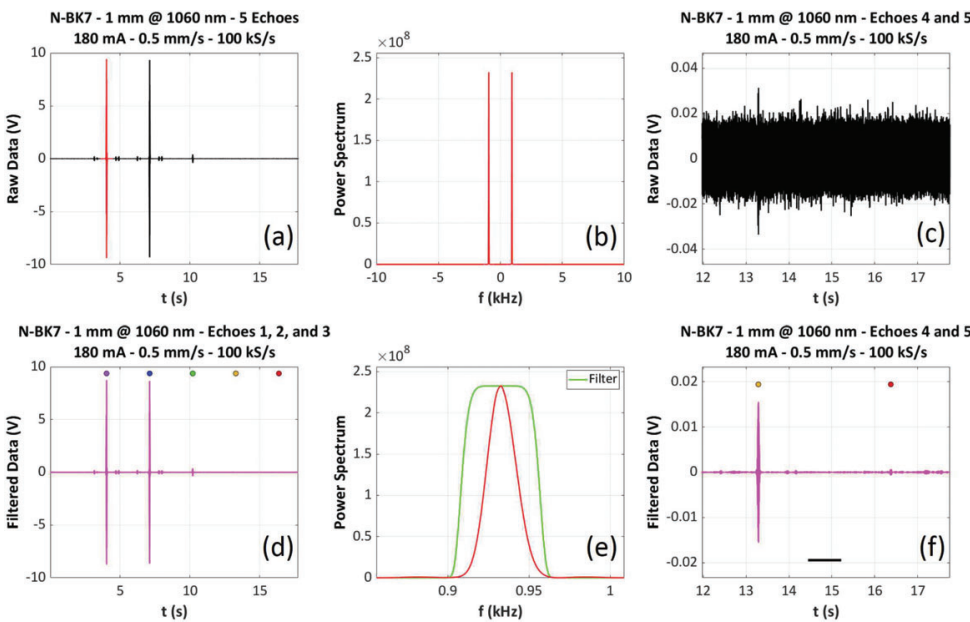

Figure 3: Backreflected light measured from $1 \mathrm{~mm}$ thick N-BK7 window (see text for more details).
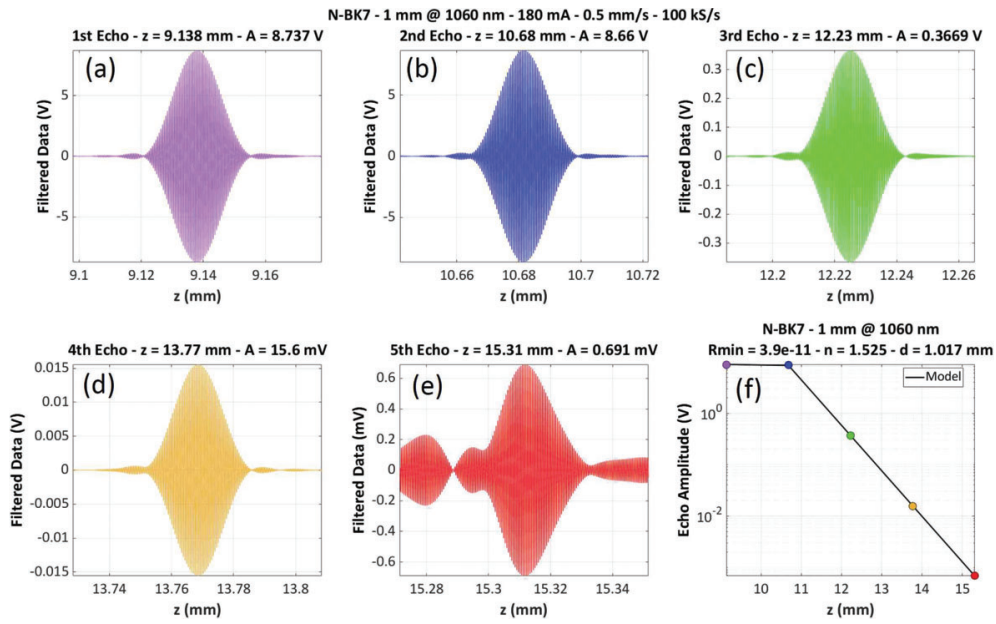

Figure 4: 4(a) to 4(e): Enlarged views of the interferograms corresponding to the five first reflections (R1 to R5) on a 1-mm thick N-BK7 window ; in each drawing title, $\mathrm{A}$ is the maximum amplitude of the corresponding echo and $\mathrm{z}$ is the position of this maximum - 4(f): comparison between the experimental results and the amplitude and position data resulting from a modelling.

\subsection{Backreflected light from $1 \mathrm{~mm}$ thick N-BK7 window}

The interferogram recorded from a $1 \mathrm{~mm}$ thick N-BK7 window is shown in Figures 3 and 4. Figure 3(a) shows the raw signal recorded during a continuous scan of the reference arm approximately $10 \mathrm{~mm}$ long, while Figure 3(c) shows an enlarged view of the same signal corresponding to the last 8 seconds of the acquisition. Applying a fast Fourier transform (FFT) to the red part of this recorded signal (first echo) allows the intrinsic power spectrum of an echo to be calculated [Figures 3(b) and 3(e), red curves] and the characteristics to the frequency band-pass filter [Figure 3(e), green curve] to be determined (central frequency, bandwidth). This band-pass filtering is applied to the Fourier transform of the raw signal and an inverse FFT allows the time dependence of the filtered signal to be reconstructed [see Figures 3(d) and 3(f), magenta curves). One can see the big improvement provided by this band-pass filtering by comparing Figures 3(c) and 3(f). Moreover, on Figures 3(d) and 3(f), colored dots are used to indicate the theoretical position of the center of the interferograms corresponding to the multiple reflections on the N-BK7 window: the agreement with the experimental results is really excellent.

Figures 4(a) to 4(e) shows an enlarged view of the interferograms corresponding to the first five echoes recorded on the 1-mm thick N-BK7 window (filtered data). One can note the very nice and almost identical shape of the 
first four echoes, and the presence of some spurious contributions in that of the fifth echo: this is induced by the low signal to noise ratio obtained when the corresponding coefficient of reflection is as low as $2.6 \times 10^{-10}$. Figure $4(\mathrm{f})$ shows a comparison between the experimental amplitude and position of the first five echoes (colored dots) and the result of a theoretical modelling (black curve): one can note the impressive quality of the agreement. Using all these results allows the minimal value of the back-reflected coefficient (signal equal to 3 times the standard deviation of the noise) to be determined, i.e. $3.9 \times 10^{-11}(-104 \mathrm{~dB})$.

\subsection{Backscattered light from a Silver mirror}
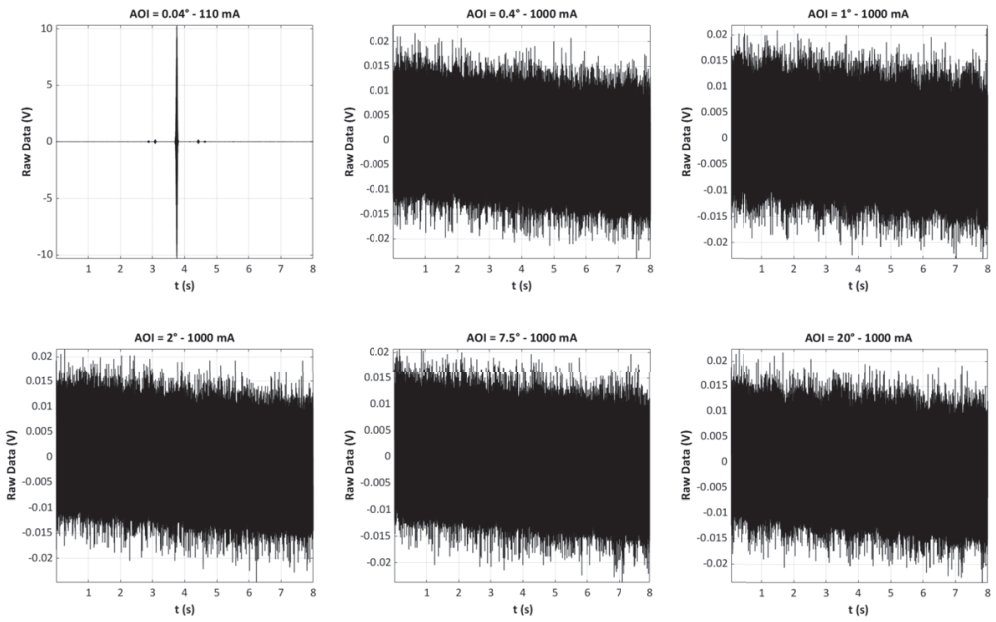

Figure 5: Back-scattered light interferograms recorded at different angles of incidence from a silver mirror (raw data) - Only a few interferograms are shown for the sake of simplicity.
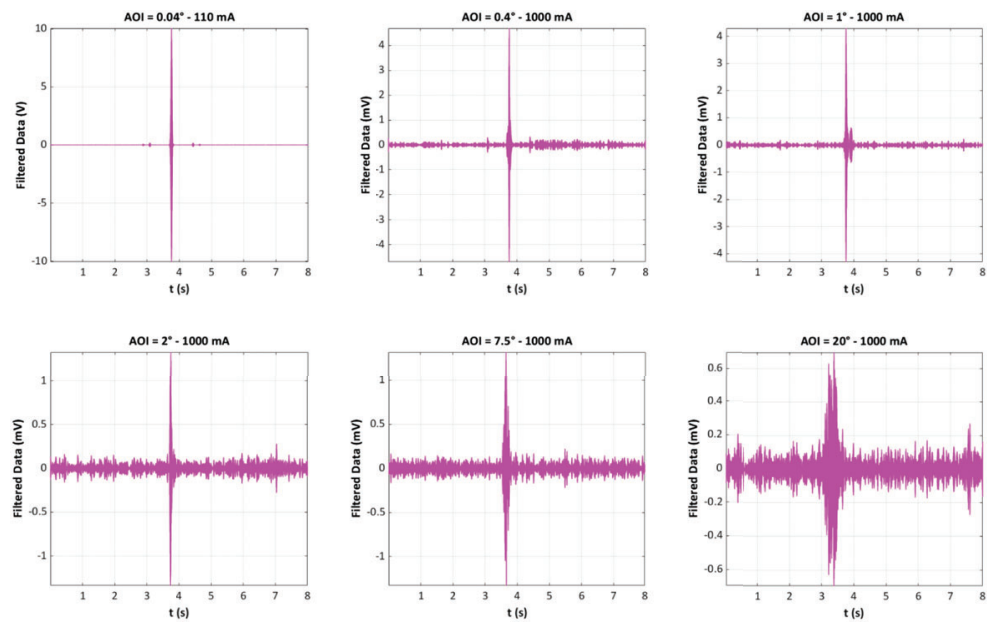

Figure 6: Same recordings as shown in Figure 5, but after frequency filtering.

In order to demonstrate the ability of the set-up to efficiently detect the light back-scattered by an optical surface, we first used a silver mirror as trial sample (reflection coefficient about $95 \%$ ). Indeed, the amount of light scattered at low tilt angle is proportional to the reflection coefficient of the sample. As the coefficient of reflection of the silver mirror is very high, the recording of the interferogram obtained at zero AOI is achieved using a very low driving current $(\mathrm{I}=110 \mathrm{~mA})$. Then we rotated the sample in steps and recorded the interferograms at full source power $(\mathrm{I}=1000 \mathrm{~mA})$. The results are shown in Figures 5 (raw data) and 6 (filtered data). In Figure 7 (a), one can see the change in position of the backscattered light interferogram versus the AOI in (blue dots), as well 

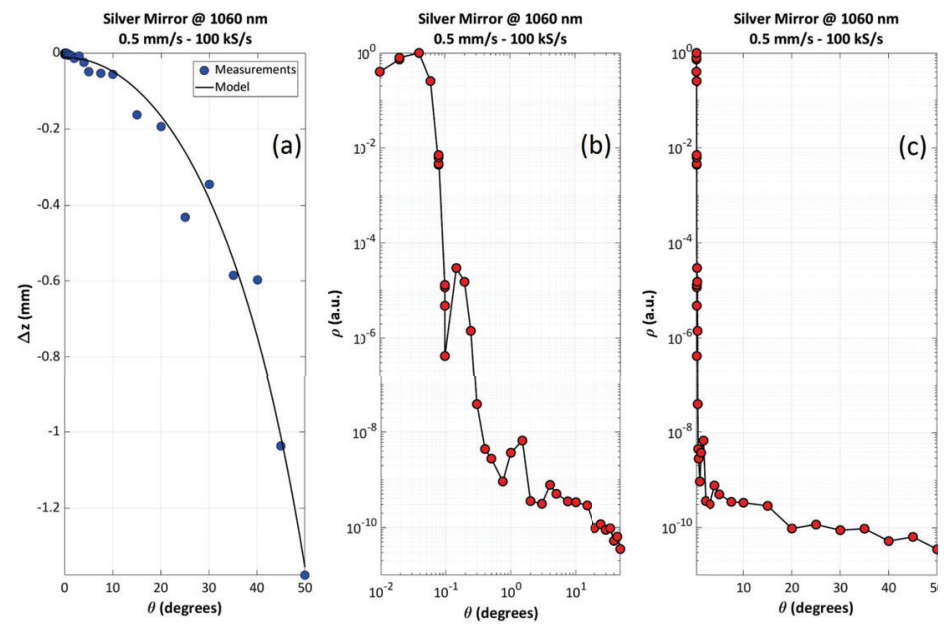

Figure 7: Backscattered light measured from Silver mirror at different AOIs. Figure 7(a) change in interferogram position versus the AOI, Figure 7(b) amplitude of the backscattered light versus AOI (log-log scale) and Figure 7(c) amplitude of the backscattered light versus AOI (log-linear scale).

as the result of a modelling (black curve) taking into account an offset $r$ between the front face of the sample and the axis of rotation. Such an offset $r$ yields a change $\Delta z$ in the interferogram position for the changing AOI described by the following relationship

$$
\Delta z=\frac{r}{\cos \theta}-r
$$

Figures 7(b) and 7(c) show the variation of the amount of light backscattered by the silver mirror versus the tilt angle of the sample $(\theta)$. The data shown in these two curves are identical, the only difference is the use of a $\log$ scale for the angle of incidence in Figure 7(b) instead of a linear scale for the same quantity in Figure 7(c). This allows to have a better view of the steep variation of this backscattered light below 0.5 degree. The amount of backscattered light is detected by the experimental set-up up to 50 degrees AOI (coefficient of reflection of around a few $\left.10^{-11}\right)$.

\subsection{Backscattered light from $1 \mathrm{~mm}$ thick N-BK7 window}
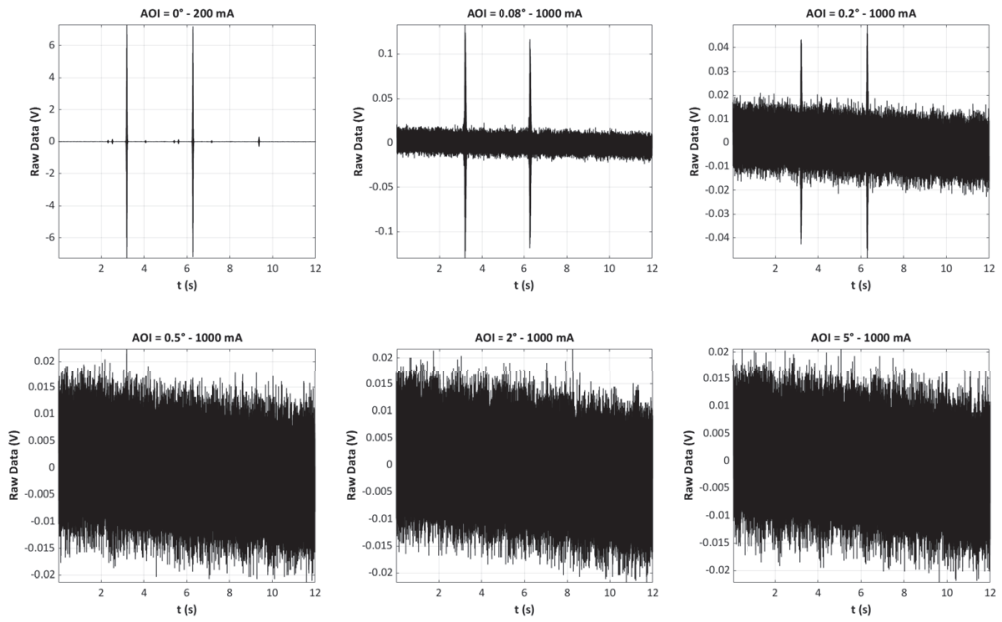

Figure 8: Back-scattered light interferograms recorded at different angles of incidence from a bare N-BK7 window (raw data) - Only a few interferograms are shown for the sake of simplicity. 

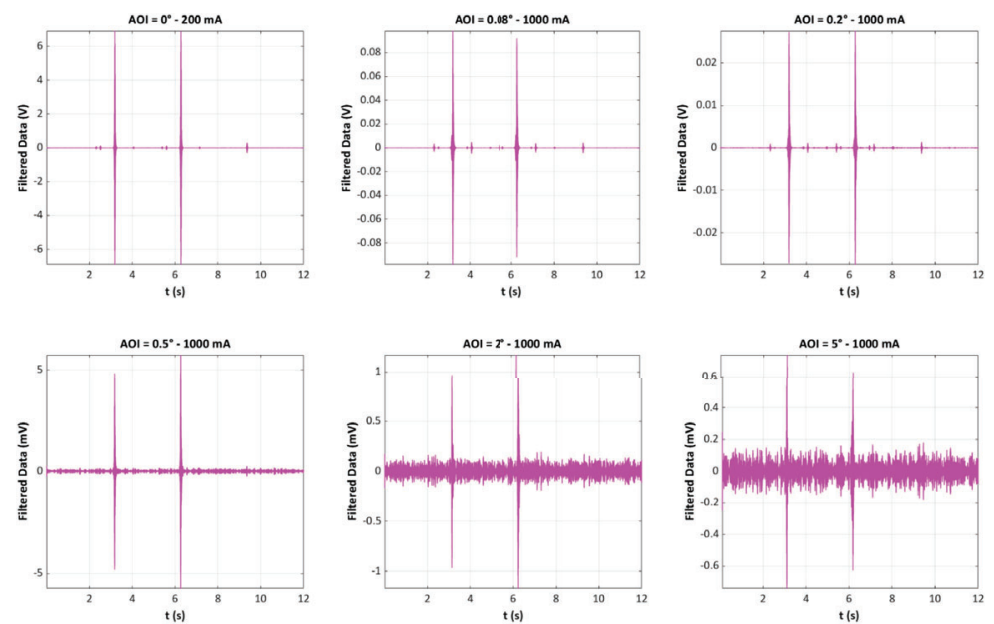

Figure 9: Same recordings as shown in Figure 8, but after frequency filtering.
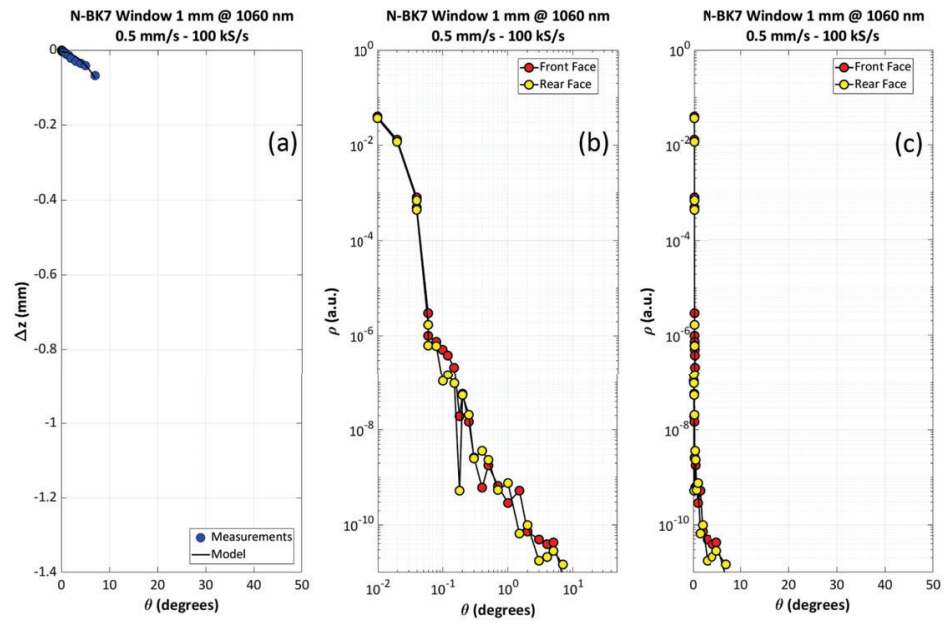

Figure 10: Backscattered light measured from N-BK7 window at different AOIs. Figure 10(a) change in interferogram position versus the AOI, Figure 10(b) amplitude of the backscattered light versus AOI (log-log scale) and Figure 10(c) amplitude of the backscattered light versus AOI (log-linear scale).

And finally, we measured light backscattered from a $1 \mathrm{~mm}$ thick N-BK7 window. Compared to the Silver mirror, the measurement is more demanding, because in this case the coefficient of reflection is only $4 \%$. Figures 8,9 , and 10 show the experimental results obtained on this bare window, using the same approach as used for the silver mirror in Figures 5, 6 and 7. One can note that the method allows the light backscattered by the front face and the rear face to be independently recorded. This property is very important for stray light metrology application because the contributions of different surfaces can be separated. One can note that the maximum $\mathrm{AOI}$ is here equal to 8 degrees as compared to 50 degrees for Silver mirror and this is due to the reduction of coefficient of reflection from $95 \%$ to $4 \%$.

\section{CONCLUSION}

We presented the preliminary results of measurement of light backscattered by a N-BK7 window and a Silver mirror using low coherence interferometry. To the best of our knowledge, the simultaneous measurement of the light backscattered from the front and rear faces of an optical window has been achieved for the very first time. The noise floor of our method is about $10^{-11}$ in terms of coefficient of reflection, and some changes in the set-up are currently underway to improve this performance up to $10^{-13}$. 


\section{ACKNOWLEDGEMENT}

This work is part of the StrayLight Working Group for the Laser Instrument Group of the LISA Consortium. The authors would like to thank the AMIdex Foundation, Aix Marseille University and the French National Space Center (CNES) for funding the research activity.

\section{References}

[1] The LIGO and Virgo scientific collaboration, "Observation of gravitational waves from a binary black hole merger," Phys. Rev. Lett. 116, 061102 (Feb 2016).

[2] A. Abramovici et al., "Ligo: The laser interferometer gravitational-wave observatory," Science 256(5055), 325-333 (1992).

[3] F. Acernese et al., "Status of virgo detector," Classical and Quantum Gravity 24, S381-S388 (sep 2007).

[4] R. Abbott et al. (LIGO Scientific Collaboration and Virgo Collaboration), "Population Properties of Compact Objects from the Second LIGO-Virgo Gravitational-Wave Transient Catalog," arXiv e-prints , arXiv:2010.14533 (Oct. 2020).

[5] R. Abbott et al., "GWTC-2: Compact Binary Coalescences Observed by LIGO and Virgo During the First Half of the Third Observing Run," arXiv e-prints , arXiv:2010.14527 (Oct. 2020).

[6] R. Abbott et al., "Open data from the first and second observing runs of advanced ligo and advanced virgo," SoftwareX 13, 100658 (2021).

[7] R. Abbott et al. (LIGO Scientific Collaboration and Virgo Collaboration), "Gw190521: A binary black hole merger with a total mass of $150 M_{\odot}, "$ Phys. Rev. Lett. 125, 101102 (Sep 2020).

[8] G. Heinzel and C. Braxmaier et al., "Successful testing of the LISA technology package (LTP) interferometer engineering model," Classical and Quantum Gravity 22, S149-S154 (apr 2005).

[9] eLISA Consortium and P. Amaro Seoane et al., "The Gravitational Universe," arXiv e-prints , arXiv:1305.5720 (May 2013).

[10] S. Barke and Y. Wang et al., "Towards a gravitational wave observatory designer: sensitivity limits of spaceborne detectors," Classical and Quantum Gravity 32, 095004 (apr 2015).

[11] S. Hild, Beyond the first Generation: Extending the Science Range of the Gravitational Wave Detector GEO600, PhD thesis, Von der Fakultat fur Mathematik und Physik der Gottfried Wilhelm Leibniz Universitat Hannover zur Erlangung des Grades (2007).

[12] D. J. Ottaway, P. Fritschel, and S. J. Waldman, "Impact of upconverted scattered light on advanced interferometric gravitational wave detectors," Opt. Express 20, 8329-8336 (Apr 2012).

[13] M. Lequime, V. Khodnevych, M. Zerrad, M. Lintz, and C. Amra, "Coherent detection of the light backscattered by a rough surface," in [Optical Interference Coatings Conference (OIC) 2019], Optical Interference Coatings Conference (OIC) 2019, TE.7, Optical Society of America (2019).

[14] V. Khodnevych, S. Di Pace, J. Y. Vinet, N. Dinu-Jaeger, and M. Lintz, "Study of the coherent perturbation of a Michelson interferometer due to the return from a scattering surface," in [International Conference on Space Optics Emdash; ICSO 2018], Society of Photo-Optical Instrumentation Engineers (SPIE) Conference Series 11180, 111807T (July 2019).

[15] K. Freischlad, "Sub-angstrom surface metrology with a virtual reference interferometer," in [Interferometry XVI: Techniques and Analysis], Schmit, J., Creath, K., Towers, C. E., and Burke, J., eds., 8493, 86 - 99, International Society for Optics and Photonics, SPIE (2012).

[16] M. Lequime and J. Lumeau, "Accurate determination of the optical performances of antireflective coatings by low coherence reflectometry," Appl. Opt. 46, 5635-5644 (Aug 2007). 